\title{
Computational Concerns in the Integration of Unmanned Airborne Systems into Controlled Airspace
}

\author{
C.W. Johnson, \\ Department of Computing Science, University of Glasgow, Scotland. \\ johnson@dcs.gla.ac.uk
}

\begin{abstract}
Unmanned Airborne Systems (UAS) offer significant benefits for long duration missions. They can also be used in situations where it is inappropriate to expose aircrew to increased levels of risk. Partly in consequence, they continue to experience accident rates that are significantly higher than those for most conventional aircraft. It can also be argued that increased mishap rates are due to lower standards of design and maintenance. UAS are, therefore, largely confined to segregated areas that are well away from other airspace users. There are growing commercial and political pressures for them to be integrated into airspace that is directly under the control of air traffic management. Police agencies would like to deploy miniature UAS in populated areas, for example, to augment conventional helicopter operations. There are proposals to operate unmanned freight operations from existing airports. Longer-term proposals include the use of UAS technology to replace the co-pilot. Automated systems might intervene only if the single human pilot is incapacitated. The following pages focus on the computational issues that form one part of wider set of 'system safety' concerns that must be addressed before UAS operations can be integrated into controlled airspace.
\end{abstract}

Keywords: UAV, UAS, Air Traffic Management, Safety, Controlled Airspace.

\section{Introduction}

The next 20 years will see enormous changes in air traffic. One aspect of this is the probable introduction of Unmanned Airborne Systems (UAS) into controlled air space. Traditionally, these aircraft have been segregated away from other airspace users in areas that are not directly under the control of Air Navigation Service Providers (ANSPs). However, a range of commercial and political pressures are acting to challenge existing safeguards. Within the US military funding for Unmanned Airborne Vehicles (UAVs) development has increased from \$3 billion in the early 1990s to over $\$ 12$ billion for 2004-2009 [1]. The civil UAS market could reach $€ 120$ million annually by 2011 . 
UAVs offer numerous benefits. They support long duration missions that would be difficult, if not impossible, to resource using conventional aircraft. Examples include the monitoring work being undertaken by the Customs and Border Patrol on the USMexico border. They also include more speculative proposals to incorporate UAVs into the security systems for the 2012 London Olympics. A new generation of unmanned freight vehicles is being deployed to help the US military avoid the Improvised Explosive Devices that are being deployed against coalition forces in Iraq and Afghanistan. Civil versions are (arguably) less than a decade away from production [2].

UAS also raise a number of concerns. They have a significantly higher accident rate than conventional aircraft [3]. Some of these mishaps stem from the human factors issues associated with maintaining remote situation awareness. It is also apparent from previous incidents that the standards that are used in the engineering of UAV platforms often fall below those required in conventional aircraft. Documents such as the Federal Aviation Administration's (FAA) 08-01: Unmanned Aircraft Systems Operations in the U.S. National Airspace System (NAS) [4], as well as EUROCONTROL's Spec-0102 on the Use of Military Unmanned Aerial Vehicles as Operational Air Traffic outside Segregated Airspace [5] and the UK Civil Aviation Authority's CAP 722 [6], therefore, place strict limits on the operation of UAS.

The commercial opportunities created by UAS make it difficult to defend these restrictions in the long run. ANSPs and regulators face growing political pressure to allow the integration of UAS operations with other airspace users [2]. CAP722, cited above, recognizes this; "While UAV flights beyond the limits of visual control ... are currently restricted to segregated airspace, the ultimate aim is to develop a regulatory framework which will enable the full integration of UAS activities with manned operations throughout UK airspace." In order for this to happen, UAV operations must not increase the risk to other airspace users. The CAA also argue that Air Traffic Management (ATM) procedures for interactive with UAS should mirror those applicable to manned aircraft and that the provision of air traffic services to UAVs should be transparent to controllers $[1,7]$.

The following pages focus on early-stage planning for the integration of UAS into controlled airspace. The computational hazards of UAV operations form part of a range of 'systemic' safety concerns. These range from the difficulties that Air Traffic Control Officers (ATCOs) experience when trying to identify who is commanding UAS operations over distributed communications systems to the problems associated with semi-autonomous operations, for example during the execution of lost link profiles, described below.

\section{Airworthiness Concerns}

UAV platforms must, typically, meet the same airworthiness requirements as conventional aircraft. For instance, Section 6 of FAA Interim Guidance 08-01 stipulates that all UAS must be in a fit state to conduct operations in the NAS. In particular, there is a requirement that the components of the system be maintained and conform to "the same airworthiness standards as defined for the 14 Code of Federal 
Regulations parts under which UAS are intended to be operated". In Europe, UAVs over $150 \mathrm{~kg}$ must have an airworthiness certificate provided by EASA. Platforms below that mass can operate under national airworthiness certification. In both cases, the approach tends to follow that outlined within FAA 08-01. UK CAA CAP722 guidance states that "If the UAS industry is to produce UAS capable of operating in all classes of airspace, it is essential... that they demonstrate equivalence to the regulatory and airworthiness standards that are set for manned aircraft".

Civil and military accident investigations have shown that airworthiness requirements are not always met by existing UAS operations [1, 2, and 9]. For example, a Canadian UAV was lost while the ground crew struggled with the engine management software in order to generate sufficient power to sustain flight. A lean mixture preset screw was set incorrectly, probably during routine maintenance. This led to a gradual fall in power during subsequent flights. The power reduction was not observed by the operating and maintenance teams until the day of the accident. Although the manufacturer's service bulletins described different launch profiles, they did not consider engine management issues to the level of detail that characterises most conventional aircraft bulletins [9].

The same UAV design was involved in a further accident when its parachute retrieval system failed to fully deploy following a strong gust of wind. The avionics software exacerbated this failure because it would not deploy the airbags that protected the vehicle against hard landings until it had sensed the deployment of the parachute. Software upgrades and operational changes were introduced to address the cause of these accidents. They arguably illustrate illustrate a 'fly-fix-fly' approach to UAS airworthiness that would not be acceptable in other forms of aviation.

Airworthiness concerns are compounded by a loss of first-person liability. In other words, it is easier to maintain meticulous standards in maintenance and operation when an aircrew's life is at risk. There are other potential explanations. For example, many UAV airframes use innovative designs in terms of their aerodynamics, avionics and ground control software. We have less experience in maintaining these platforms, compared to the long history of maintenance procedures for conventional aircraft. Other concerns relate to the difficulty of communicating maintenance information and software upgrades given that UAS are operated in many different areas around the globe. It can also be difficult for the operational teams to provide maintenance engineers with information about particular problems given that they, in turn, have to rely on limited information feeds provided to the Ground Control Systems.

Political pressures can also compromise airworthiness requirements. For instance, the NTSB report into the loss of the US Customs and Border Patrol's (CBP) Predator near Nogales Arizona found that "At the time of the accident, CBP was unable to certify to the FAA that BP-101 was airworthy. Because of national security issues and past experience with similar UASs, the FAA temporarily waived this requirement for the issuance of the Certificate of Waiver or Authorization (COA) to operate in the National Airspace System (NAS)". Section 6 of FAA 08-01 was not enforced because the CBP had been directed to start flying the Predator programme at short notice. There was political pressure to deploy UAVs as a means of reducing illegal crossborder immigration [2]. 
Airworthiness concerns affect all aspects of UAS operations from avionics to ground control software. They also have a secondary impact by increasing the workload of ground crews and, in the future, may place significant perceptual and cognitive burdens on air traffic controllers. Such preoccupations erode safety margins and can undermine the situation awareness of ATM personnel and ground control teams. It is critical that any future studies into the integration of UAVs build on these observations by adopting a systems perspective that considers these knock-on effects, for example, on ATCOs' ability to operate complex computation infrastructures, including conventional communications systems, Medium Term Conflict Detection tools etc, while also responding to the UAS failure.

\section{Radar Profiles and Transponders}

A key requirement for the integration of UAVs into controlled airspace is that they must be 'visible' to the computational systems used by Air Traffic Management. This is particularly important given the anticipated role of 4D trajectory software systems within both the European SESAR and US NextGen initiatives. Many UAV airframes are considerably smaller than manned vehicles even though they have the potential to cause significant damage during any collision. The military heritage of these aircraft has also led to legacy designs that are deliberately hard to see on conventional radar systems. These effects are exacerbated when composite materials are used to reduce the mass of the UAV and, thereby, extend the operational duration of the platforms.

Some of these concerns can be overcome by the development of lightweight transponders and Automatic Dependent Surveillance-Broadcast (ADS-B) avionics that transmit identification and trajectory information. However, this is not a panacea. During the Nogales crash, mentioned above, the Predator's avionics software responded to a loss of communications with the Ground Control System by reducing power requirements. This was intended to extend the range of the UAV as it flew to an area where contact might be resumed. The transponder was one of the first systems to be disabled as a means of conserving power. The software, therefore, removed potential identification information at a time when Air Traffic Management personnel had the greatest need to locate the UAV.

A recent series of simulator studies have identified additional concerns that arise from the integration of UAS, even when transponder information is available [7]. Vogt and Udovic connected a Mission Management System (MMS) for a search task to a high altitude, high endurance (HALE) UAS simulator. This was then linked to an air traffic control simulator. Eight different traffic scenarios were studied for seven emergency situations. These included the combined failure of radio communication and data link connections to the UAV. Their results showed that ATCOs could cope with these failures in civil controlled airspace using current procedures. However, the ATCOs in the study argued that the transponder Emergency Squawk 7700 was not suitable for UAS data link losses. In this situation, the UAV is still fully operational and not necessarily in an emergency situation. They, therefore, proposed that a new unique transponder code would help ATM staff to identify this failure mode in autonomous flight. Vogt and Udovic's work is important because it provides a more 
optimistic view of UAS integration than the stance adopted by this paper. The divergence of views may, in part, be due to their use of simulation studies compared to the accident and incident data that motivates our work. It seems likely that the experience of future integration may lie somewhere between our alternate perspectives.

\section{On-Board Software Verification and Certified FMS}

UAV development costs have, typically, been lower than those of conventional aircraft. However, future integration requires that on-board avionics are of a comparable standard to those used in conventional aircraft. The FAA recognises that the testing of on-board software remains one of the biggest hurdles to the integration of UAS operations into controlled airspace. It is for this reason that they have entered into cooperative research and development agreements (CRDA) with commercial organizations to evaluate the integration of existing Flight Management Systems (FMS) into UAV platforms. The first flights began in December 2009. The purpose of this work is to demonstrate that a pre-certified system could be used within these unmanned platforms, significantly reducing the costs associated with the approval of a new UAV-specific FMS. During the initial test flights, the ground crew performed lateral path guidance to airways, flew direct to waypoints and issued airspeed and altitude commands during climb, cruise and descent. This established the potential use of the FMS to implement ATM commands in real-time trajectory negotiation. The immediate objectives for the next phase are to introduce 4D FMS control systems into FAA simulations of the National Airspace System (NAS). These simulations will be similar to those conducted by Vogt and Udovic [7].

The relative speed of innovation is a key issue. The re-use of an existing commercial FMS provides an accelerated route towards UAV integration. It is less easy to interpret the possible consequences for overall systems safety [8]. On the one hand, the application of a pre-certified FMS can be argued to free analytical resources to identify systems level risks. Commercial and regulatory bodies can re-task the teams that would otherwise have been used on UAS FMS certification to consider the wider hazards of integrated operations. A more sombre interpretation of the accelerated timetable might conclude that political and commercial pressures are working to ensure the rapid integration of reduced crew, freight operations into controlled air space.

The introduction of new technology also creates novel demands for software testing. For example, previous attention on the role of software in the integration of UAS into controlled air space has focuses on the development of advanced 'sense and avoid' techniques [1]. These systems use data fusion from a range of airborne systems to first identify and then respond to potential conflicts with other airspace users, in the same way that a pilot might respond to an Airborne Collision Avoidance System (ACAS) warning. It is difficult to identify appropriate verification and validation criteria for these future sensing technologies, especially given potential interactions between multiple UAVs with conventional aircraft. 


\section{Lost Link Profiles}

The autonomous operation of lost link software raises further concerns for flight safety. Most UAS enable their operators to pre-program a number of waypoints that define a lost-link profile. This forms the route that the vehicle will fly should it loose contact with the Ground Control System. Many UAVs provide the ability to programme and store a number of these trajectories. The ground team must, therefore, nominate the most appropriate recovery route for the particular mission that they are about to conduct. The 'active' lost link profile can also be changed during a mission as operational requirements lead the UAV away from its intended flight path.

The intention behind the creation and maintenance of pre-programmed lost link profiles is that the vehicle will autonomously navigate to a holding position where recovery teams can attempt to restore communications. In the worst case, these profiles indicate the bounds within which the UAV should come down when its power reserves are exhausted. FAA 08-01 requires that "In all cases, the UAS must be provided with a means of automatic recovery in the event of a lost link. There are many acceptable approaches to satisfy the requirement. The intent is to ensure airborne operations are predictable in the event of lost link...It is the applicant's responsibility to demonstrate that injury to persons or property along the flight path is extremely improbable... UA with performance characteristics that impede normal air traffic operations may be restricted in their operations". Similarly, UAS operations should avoid routes with heavy traffic or with open assemblies of people. These can only be approved in emergency or relief situations if 'the proposed mitigation strategies are found to be acceptable'. This requires that applicants conduct a formal risk assessment with associated safety argument to demonstrate that the residual hazards are "extremely improbable".

Experience has illustrated the practical difficulties that can arise when trying to meet lost-link safety requirements. For instance, the crew of the Nogales Predator were uncertain about its likely flight-path after communications were lost. There was a concern that it might crash on the route from its last known operating position to the first of the lost link waypoints. In the event, the UAV descended from segregated airspace into controlled airspace creating a significant hazard as it autonomously navigated towards its rendezvous. This incident demonstrates that unless greater care is taken to update and maintain pre-programmed lost-link profiles then there is a considerable danger for adjacent ATM operations even while UAVs operate in segregated airspace.

\section{Ground Control Software Reliability Issues}

Concerns over the role of computational systems in the integration of UAS into controlled airspace must extend beyond the airborne systems to also include the design, operation and maintenance of Ground Control Systems (GCSs). These applications provide the crucial link between the operating staff and the airborne systems. In addition to reliability concerns, there is a lack of standardisation both in 
terms of the user interfaces but also in terms of the training that different crews have when they operate ground control software.

Previous sections have identified the lack of 'first person liability' and 'corporate responsibility' that combined with the experimental nature of UAV operations to explain some safety concerns. Previous accident reports have also shown that poorly documented maintenance procedures and inadequate reviews of corrective actions have undermined the reliability of UAS Ground Control Systems [1]. For instance, several of the losses documented in our previous studies have been triggered by bugs that cause the ground control software to 'lockup' [2, 9, and 10]. In one instance, a subsequent review of the GCS software logs showed that there had been nine previous lockups in the three months before the loss of the UAV. Two of these occurred before launch on the day of the accident. The ground team had developed an informal practice of correcting these failures by cycling the power on the frozen working position. Although this addressed the immediate symptoms of the problem, it did not identify longer term solutions nor did it provide any assurance that recycling the power avoided undesirable side-effects on the GCS configuration.

UAS, typically, exploit redundancy as a primary means of improving the reliability of Ground Control Systems. There are typically two or more workstations that can be reconfigured to support a number of roles. For instance, many UAVs distinguish between the Mission Planner who coordinates current and future operations and reports to outside agencies; the Air Vehicle Operator who controls and monitors the vehicle and the Payload Operator who performs similar functions for the imaging equipment. The Mission Planner and Air Vehicle Operator workstations are usually identical and provide additional redundancy in the case of failure. However, this redundancy is not always exploited in the ways envisaged by UAS suppliers. Another accident occurred when the processor board of one working position was simply exchanged with that of a neighbouring 'frozen' position [1]. Although this provided a temporary fix, the second position subsequently suffered the same hardware problems that had affected the first workstation.

\section{Human Factors, SOPs and Situation Awareness}

Doubts remain over the short term feasibility of the 'sense and avoid' techniques required to support autonomous operation. In consequence, it seems likely that UAS will continue to rely upon ground based operators. This creates enormous difficulties. It is extremely hard to create and maintain a high degree of remote situation awareness during UAS operations. This can be illustrated by the issues that arise in determining an optimum allocation of on-board sensing systems during different phases of flight. A recent accident occurred when the UAV was circling a potential landing site. The Payload Operator had skewed their camera at 90 degrees to acquire the recovery area. They did not see the mountain before the UAV flew into it. The loss of visual situation awareness was compounded because they crew had set the automated altitude warning at 200 rather than $300 \mathrm{~m}$ AGL. This reduced the number of spurious alarms that were generated during routine flights in mountainous terrain. It also delayed the automated alarm so that the crew were only warned of a potential collision immediately before the accident. The large number of spurious alarms may 
also explain why the crew habitually ignored the aural warning associated with the altitude alarm. Situation awareness was also compromised by the crew's decision to display engine monitoring information on their workstations rather than the altitude screen that might have shown the potential danger from rising terrain.

In most other areas of aviation, Standard Operating Procedures (SOPs) would have provided guidance on the appropriate use of the software systems that are intended to support situation awareness. However, the crew involved in this accident did not have access to detailed SOPs. As in many other areas of UAS operations, the impetus to achieve operational benefits often overrides necessary caution. The crew also lacked a Standard Manoeuvre Manual, crew standard procedures, standard crew terminology etc. These factors combined to create the context in which it is hardly surprising that they suffered such an extreme loss of orientation on their approach to the landing zone. The lack of SOPs and associated documentation also creates problems for ATCOs who must monitor and support any eventual integration into controlled airspace. Air Traffic Management personnel cannot assume that similar UAS control teams will respond in similar ways to similar situations unless they have been trained to use similar operating procedures.

Even when SOPs have been drafted to govern the operation of UAS, there is no guarantee that crews can maintain the level of situation awareness that is necessary to support interaction both with conventional aircrews and with ATM organisations [10]. In particular, it can be difficult to draft procedures that govern every possible contingency that might arise during the operation of a UAV. These include the failures of on-board avionics software but also changing meteorological conditions as well as the changing demands of particular missions. Any one of these factors can undermine situation awareness. Further problems arise from our very limited experience of UAS applications even in segregated airspace.

\section{ATM Software Integration: Safety Nets}

The integration of UAVs into controlled air space has particular importance for the configuration and operation of safety net applications. These are used to alert ATCOs or pilots to potential hazards with sufficient time for an adverse situation to be resolved. Ground-based safety nets exploit both radar and ADS-B surveillance data. These include the short term alerting systems that provide warnings times up to two minutes before an adverse event. Additional work would also be required to reconfigure medium term altering systems, which tend to operate up to 20 minutes before a potential incident. Area Proximity Warning (APW) systems alert ATM staff to the potential infringement of restricted airspace volumes. Similarly, Minimum Safe Altitude Warnings (MSAW) are issued when aircraft descend below pre-specified 'safe' altitudes. Approach Path Monitors (APM) will warn ATCOs about deviations from a pre-specified glide path. The integration of UAV's into controlled airspace poses particular problems for the operation of these software applications because these vehicles are difficult to track using conventional surveillance techniques. In consequence, the existing ground based safety nets may not function to the level of reliability that is assumed for existing aircraft. Further 
problems arise because increasing the sensitivity of surveillance equipment is likely to increase the number of false alarms.

In contrast, airborne safety nets warn the crews of conventional aircraft about potentially hazardous situations. Some of these provide warnings about on-board hazards, including fires or hull depressurisation and have an indirect impact on Air Traffic management. However, other airborne safety nets provide stall warnings, Ground Proximity Warnings (GPWS) and Airborne Collision Avoidance warnings (ACAS). ACAS is particularly significant in this respect as the $\square$ berlingen accident has illustrated the consequences that can arise with on-board warnings contradict the clearances provided by ATC [11]. It is difficult to predict the impact that a UAV separation infringement might have on the crews of multiple conventional aircraft that would be alerted by airborne safety nets.

\section{ATM Software Integration: Ground Movement Systems}

Ground movement systems represent one of the most neglected areas of concern over the integration of UAVs into controlled airspace. Plans for future UAS freight operations, typically, assume that it will be possible to land these aircraft at existing airports. The economic arguments depend upon the integration of UAV cargo deliveries with existing ground-based distribution networks from regional aerodromes. This creates potential problems both for approach and airport control. The Linate runway incursion shows the increased workload that arises when ATCOs synchronise heterogeneous traffic movements [12]. In this accident, ATM personnel had to coordinate large passenger aircraft with the requirements of general aviation and of small commuter jets on the same runway infrastructures. The causes of the collision were exacerbated by communications problems between the ATCOs and the aircrews. Unless considerable care is taken then there is a danger that many of these existing concerns over the safety of ground movement will be exacerbated as ATM personnel coordinate conventional and unmanned operations.

One aspect of this integration between UAVs and ground movement operations is the ability to use Surface Movement Radar (SMR) systems. These software tools present ground controllers with real time updates on the location of aircraft and vehicles. They are intended to augment direct visual observations from the Control Tower and are particularly useful in poor weather or when ATM personnel have restricted views, for instance, following alterations to piers etc. However, SMRs also suffer from the reduced radar profile and composite fabrication of UAVs that create problems for airborne surveillance. During initial trials, it has been very difficult o distinguish UAS movements from those of ground vehicles. In consequence both the FAA and UK ANSP have begun studies to upgrade SMR as a means of helping Tower staff to monitor UAVs.

The challenges posed by UAS ground movements are not simply related to the software and hardware technologies that are used to support Air Traffic Management. Remote modes of operation and control create entirely new hazards. For example, in the past it has been possible to provide redundant communications infrastructures that ATCOs can use to make direct contact with aircrews if the primary ATM transmitter is not available. In the future, this will not be sufficient when the UAS GCS team 
may be many thousands of miles beyond the range of any transmitters that are directly under the control of the ANSP. The meta level point here is that the integration of UAVs will force 'root and branch' changes to the safety arguments and risk assessments that were previously developed to demonstrate that ATM operations were acceptably safe.

\section{Longitudinal UAV GCS Crew Interaction}

It is relatively straightforward to integrate models of UAV operations into existing ATM training simulators [7]. However, it can be hard to recreate a representative sample of traffic patterns or to identify the operating procedures that might be introduced to support UAS integration. For instance, we do not know how UAV GCS crews will interact with long haul freight operations. In some scenarios, control may be handled from a single remote base. In other prototypes, control can be transferred in flight from a GCS at the point of origin to a second team handling the arrival and processing of the UAV at the destination airport. These different control models have a profound impact upon the workload both of GCS crews and also on ATM personnel. Most ANSPs lack the resources necessary to explore a broad range of possible scenarios for the integration of UASs. Hence it is difficult to ensure that we have simulated the broad range of potential hazards that might emerge from interactions between different computational systems within different categories of controlled airspace.

It is also difficult to predict the impact of UAS operations on the informal and undocumented working practices that continue to be a common feature of many Air Traffic Management organisations. These may violate recognised operating procedures. Examples include the Single Manned Operation and the informal shift handover practices that have been identified as a cause of previous accidents $[11,12]$. Many of these behaviours emerge over long periods of time as teams of co-workers develop undocumented methods of working - often in response to the demands of high levels of traffic or conversely to deal with the boredom of quiet times. It may take weeks or months before teams identify these ad hoc working practices. In this case, uncertainty about future operating practices combines with uncertainty over potential control models to undermine the use of longitudinal simulations as a means of identifying hazardous interactions between ATM systems, UAV operations and other airspace users.

\section{UAV Crew Interaction with ATCOs and other Airspace Users}

The economic arguments in favour of UAV operations often assume reduced manning levels or lower training requirements for GCS teams compared to conventional aircrews. This creates problems when ATM staff apply existing SOPs to support UAS integration with controlled airspace. Ground control teams may lack the levels of skill and expertise possessed by existing pilots. These problems are compounded when waivers are granted so that UAV crews do not even meet these reduced 
requirements. Such exemptions have contributed to the causes of several previous UAS accidents [1,2, and 9]. They create significant concerns over the ability of UAV GCS teams to operate the software systems that are provided by their ground based workstations. Further concerns surround the uncertainty that exists over the roles to be performed by members of the UAS GCS crews. On the conventional flight deck, the division of tasks and responsibilities has emerged over several decades. The roles performed by the teams who operate Ground Control Systems seem to rely on a process of trial and error. A recent military deployment began with two working positions for a Mission Planner and Air Vehicle Operator. An Air Vehicle Commander role was then added when it was realised that two-person crews lacked sufficient expertise either as a pilot or a navigator. 'Airworthiness concerns' then led to the introduction of a fourth member of the GCS teams.

The gradual development of operational roles raises concerns about the exchange of information within UAV GCS teams as well as concerns for interaction with external agencies, including Air Traffic Management. Potential communications problems can be illustrated by the lack of contact between the operators of the Nogales Predator and Air Traffic Management after the link was lost. Regulations required that the UAV pilot in charge must notify ATM of the following information after any failure in the communications link:

1. The UAS call sign.

2. UAS IFF [Identification, Friend or Foe] squawk.

3. Lost link profile.

4. Last known position.

5. Pre-programmed airspeed.

6. Usable fuel remaining (expressed in hours and minutes).

7. Heading/routing from the last known position to the lost link emergency mission loiter.

Instead, it was an ATCO who contacted the GCS crew after they lost contact with the UAV and the transponder had stopped working. The pilot did not inform them that the Predator had descended below its minimum permitted flight level (14,000 feet MSL). At this point, the pilot or the ATCO should have declared an emergency and taken measures to alert traffic in the area. They should have alerted neighbouring centres to monitor the missing vehicle. The ATC could also have started efforts to increase the level of surveillance on the UAV, for instance by contacting the Western Area Defence Sector to gather information using their height finding radar. These omissions illustrate the more general problems that arise from the understandable reluctance of a GCS crew to admit to any external agency that they have lost control of their vehicle.

Many UASs offer entirely new models of interaction. In particular, it is now possible to consider the circumstances under which an ATCO might order the destruction of a UAV without recourse to military action. The decision to destroy a vehicle in flight might be necessary if it could not be ordered off a trajectory that would take it towards a major population centre and there were intermittent communications failures. Such actions would be inconceivable for conventional aircraft. It is unclear how such an intervention would be initiated, for example 
whether it would necessarily involve consultation between the GCS crew and senior ANSP personnel or whether it could be directly commanded using future ATM workstations.

\section{Conclusions and Further Work}

Unmanned Airborne Systems (UAS) offer significant benefits. They can be operated for long duration missions by multiple teams of operators. They can also be used in situations where it would be inappropriate to risk conventional aircraft. However, UAVs continue to experience accident rates that are significantly higher than those for most conventional aircraft [3]. Partly in consequence, existing regulations segregate UAS operations away from areas under Air Traffic Management ('controlled airspace').

There are strong pressures to ease these restrictions. Police agencies would like to deploy miniature UAVs in populated areas, for example, to augment conventional helicopter operations. There are proposals to operate civil UAV freight operations from existing airports. Longer-term proposals include the use of UAV technology to replace the co-pilot and allow single crewed operation Automated systems might intervene only if the single human pilot is incapacitated.

This paper has identified a number of software-related safety concerns that arise from UAV operations within controlled airspace. In particular, we have identified the prominent role of software avionic failures in previous UAS accidents as well as issues relating to the programming of lost link profiles. We have also identified the difficulties that many UAV GCS teams face in maintaining remote situation awareness when they must simultaneously interact with ATM personnel, with other airspace users and with the remote vehicle.

We have also considered hazards from UAS integration for existing ATM software. Although much of the attention has been devoted to airborne safety nets, there are significant concerns over the ground movement systems that help ATCOs monitor and plan airport operations. The closing sections of this paper have looked at issues that cut across these different areas. For instance, there are concerns about the use of simulator studies to anticipate longer term interactions as ATCOs and GCS crews work together to integrate UAS operations with conventional traffic patterns. It seems very unlikely that the political and commercial pressures promoting UAS operations will provide sufficient time to adequately address all of these potential hazards.

\section{References}

1. Johnson, C.W., The Safety Research Challenges for the Air Traffic Management Of Unmanned Aerial Systems (UAS). In B. Kirwan (ed.), Proceedings of the 6th EUROCONTROL Experimental Centre Safety Research and Development Workshop. Held in Munich, Germany, October 21st \& 22 ${ }^{\text {nd }}$, EUROCONTROL Experimental Centre, Brètigny, France, 2009. 
2. Johnson, C.W., Insights from the Nogales Predator Crash for the Integration of UAVs into the National Airspace System under FAA Interim Operational Guidance 08-01. In J.M. Livingston, R. Barnes, D. Swallom and W. Pottraz (eds.), Proceedings of the 27th International Conference on Systems Safety, Huntsville, Alabama, USA 2009, International Systems Safety Society, Unionville, VA, USA, 3066-3076, 2009.

3. Patchett C. and Sastry V., A Preliminary Model of Accident Causality for Uninhabited Autonomous Air Systems and Its Implications for Their Decision Architectures, Proceedings of the Tenth International Conference on Computer Modelling and Simulation (UKSIM 2008), pp.487-492, 2008.

4. FAA Aviation Safety Unmanned Aircraft Program Office AIR-160, Unmanned Aircraft Systems Operations in the U. S. National Airspace System, Interim Operational Approval Guidance 08-01, Washington DC, USA, March 13, 2008.

5. EUROCONTROL Specifications For The Use Of Military Unmanned Aerial Vehicles As Operational Air Traffic Outside Segregated Airspace, Brussels, Belgium, EUROCONTROL-SPEC-0102, 26 $6^{\text {th }}$ July 2007.

6. UK Civil Aviation Authority, Directorate of Airspace Policy, Unmanned Aircraft System Operations in UK Airspace - Guidance, London, UK, $6^{\text {th }}$ April 2010. Available from http://www.caa.co.uk/docs/33/CAP722.pdf, last accessed June 2010.

7. Vogt, J. \& Udovic, A., Validation of Unmanned Aircraft Systems' (UAS) Integration into the Airspace - The VUSIL Project. German Aviation and Space Congress, September 2008, Darmstadt, Germany, 2008.

8. Williams, K.W., A Summary of Unmanned Aircraft Accident/Incident Data: Human Factors Implications. (December 2004), DOT/FAA/AM-04/24, Office of Aerospace Medicine.

9. Johnson, C.W., Act in Haste, Repent at Leisure: An Overview of Operational Incidents Involving UAVs in Afghanistan (2003-2005). In P. Casely and C.W. Johnson (eds.), Third IET Systems Safety Conference, NEC, Birmingham, UK, 2008, IET Conference Publications, Savoy Place, London, 2008.

10. Johnson, C.W. and Shea, C., The Hidden Human Factors in Unmanned Aerial Vehicles. In R.J. Simmons, D.J. Mohan and M. Mullane (eds.), Proceedings of the 26th International Conference on Systems Safety, Vancouver, Canada 2008, International Systems Safety Society, Unionville, VA, USA, 2008.

11. Johnson, C.W., Kirwan, B., Licu, T. and Statsny, P., Recognition Primed Decision Making and the Organisational Response to Accidents: $\square$ berlingen and the Challenges of Safety Improvement in European Air Traffic Management. Safety Science, (47):853-872, 2009.

12. Johnson, C.W., Linate and $\square$ berlingen: Understanding the Role that Public Policy Plays in the Failure of Air Traffic Management Systems. In C. Balducelli and S. Bologna (eds.), Proceedings of the ENEA International Workshop on Complex Networks and Infrastructure Protection, International Emergency Management Society/Italian National Agency for New Technologies, Energy and the Environment, 508-519, Rome, Italy, 2006. 